\title{
THE MECHANISM OF SELF-REVERSAL OF THERMOREMANENCE IN NATURAL HEMOILMENITE CRYSTALS: NEW EXPERIMENTAL DATA AND MODEL
}

\author{
Michel Prévot ${ }^{\mathrm{a}, *}$, Kenneth A. Hoffman ${ }^{\mathrm{b}}$, Avto Goguitchaichvilic, \\ Jean-Claude Doukhan ${ }^{d}$, Valera Shcherbakov ${ }^{e}$, Mansour Bina ${ }^{f}$
}

${ }^{a}$ Laboratoire de Géophysique, Tectonique et Sédimentologie, CNRS \& Université de Montpellier 2, 34095 Montpellier Cedex 05, France

${ }^{\mathrm{b}}$ Physics Department, California Polytechnic State University, San Luis Obispo, CA 93407, USA

${ }^{c}$ Instituto de Geofisica, Universitad Nacional Autonomia de Mexico, 04510 Mexico D.F., Mexico

${ }^{\mathrm{d}}$ Laboratoire de Structure et Propriétés de l’Etat Solide, Université des Sciences et Technologies de Lille, 59655 Villeneuve d'Ascq Cedex, France

e Geophysical Observatory 'Borok', United Institute of the Physics of the Earth, Borok, Nekouzsky region, Yaroslaskaya oblast, 152742, Russia

${ }^{\mathrm{f}}$ Laboratoire de Géomagnétisme, CNRS and IPGP, 4 avenue de Neptune, 94107 Saint-Maur Cedex, France.

* Corresponding author. E-mail: prevot@dstu.univ-montp2.fr

\begin{abstract}
New magnetic and mineralogical findings on self-reversing hemoilmenite $\left(\mathrm{Fe}_{2-\mathrm{y}} \mathrm{Ti}_{\mathrm{y}} \mathrm{O}_{3}\right)$ grains from Pinatubo lavas (1991 eruption) provide important clues regarding the acquisition process of reverse thermoremanent magnetization (rTRM) in this solid solution series. Magnetic Force Microscopy indicates the presence of multidomain magnetic structures in coexisting strongly and weakly magnetic crystallographic regions having compositions of $y \cong 0.54$ and $y \cong 0.53$, respectively. Yet, continuous thermal demagnetization of natural and laboratory TRM carried out on both whole rock samples and single hemoilmenite crystals shows that the magnitude of a normal TRM (nTRM) component, observed at temperatures above the Curie point of the strongly magnetic self-reversing regions, is much too large to be carried by a phase that is entirely cation-disordered. Consistent with this observation are findings using Transmission Electron Microscopy (TEM) which, in contrast to that what is commonly assumed, reveals the weakly magnetic regions to be magnetically heterogeneous. Specifically, these regions are found to contain tiny $(20-40 \mathrm{~nm})$ domains that are cation-ordered and evidently ferrimagnetic dispersed within the cation-disordered, presumably spin-canted antiferromagnetic matrix. Given these findings, we argue that the so-called nTRM-carrying $\mathrm{x}$-phase is itself partially cation-ordered, and thus ferrimagnetic, as postulated first by Ishikawa and Syono (1962).

We propose a "nanophase" self-reversal model for the ilmenite-hematite solid solution series in which the rTRM and nTRM components are carried by the cores and margins, respectively, of the tiny, partially cation-ordered nano-sized domains observed by TEM. Due to the partial cation order, both the core and the margin of each domain are expected to behave in a ferrimagnetic fashion at temperatures below their respective Curie points. However, given the kinetics of the ordering process, their cation distributions need be antiphase, which causes their magnetic moments to be oppositely aligned. Since it is most reasonable to consider each margin to be slightly more Fe-rich than the inside core, upon cooling the
\end{abstract}


margins acquire a magnetic remanence first (a nTRM). Then, upon further cooling, given that the intralayer and interlayer nearest-neighbor superexchange interactions are ferromagnetic and antiferromagnetic, respectively, the net magnetic moment of the core material need be oppositely aligned (producing a rTRM).

The nano-sized regions would indeed behave in a superparamagnetic (SP) fashion if magnetically uncoupled to adjacent material; however, the spins in the margins (the x-phase) must be locked through superexchange to those of the surrounding disordered matrix, which we also claim to be locally enriched in iron. If so, then the magnetization of the x-phase can be both highly-coercive and thermally stable, as observed experimentally. Upon stepwise thermal demagnetization, the self-reversed remanence measured at room temperature is not destroyed until the unblocking temperature of the disordered Fe-enriched aureole (approximately $410^{\circ} \mathrm{C}$ ) is reached. Mineralogical considerations and magnetic evidence from previous works suggest that this model is generally valid for self-reversed dacitic pumice, in particular the Mt. Haruna dacite and the 1985 Nevado del Ruiz dacitic andesite.

Key words: geophysics, paleomagnetism, rock magnetism, magnetic force microscopy, electron transmission microscopy, self-reversal, thermoremanent magnetization, exchange interaction, ilmenitehematite, dacite, pumice, Pinatubo, Philippines

\section{Introduction}

Fifty years after Louis Néel's (1951) article questioning the reality of geomagnetic reversalsbecause of the theoretical possibility for the thermoremanent magnetization (TRM) of volcanic rocks to self-reverse during cooling - the exact mechanism of this phenomenon remains a matter of debate. Following the comprehensive work of Uyeda $(1957,1958)$, it became clear that this unusual property is an intrinsic characteristic of hemoilmenite $\left(\mathrm{Fe}_{2-\mathrm{y}} \mathrm{Ti}_{\mathrm{y}} \mathrm{O}_{3}\right)$ with $0.51<\mathrm{y}<0.73$ (Westcott-Lewis and Parry, 1971b), and in some manner a consequence of exchange interactions acting between coexisting phases that had resulted from an incomplete cation order-disorder transformation. As pointed out by Uyeda, the first step of this thermoremanent process is the recording of the correct field direction (and, hence, correct polarity) by a magnetic phase so weak as to elude magnetic detection. Ishikawa and Syono (1962, 1963), who later offered their complete model of the self-reversal process, named this weakly magnetic phase the " $\mathrm{x}$ phase.” Regardless of the precise compositional nature of the x-phase and the manner in which it is developed in a hemoilmenite crystal, it must evidently have a Curie temperature higher than that of the strongly magnetic phase that ultimately carries the self-reversed TRM. The following two models, based on the work by Uyeda (1958) and by Ishikawa and Syono (1963), respectively, are both based on magnetic (and sometimes mineralogical) observations of synthetic crystals, yet assume different physical attributes to the $x$ phase:

Model 1 (two-phase model). In the initial version of this model, proposed first by Uyeda (1958), xphase regions are the relics of once homogeneous cation disordered material following partial cation ordering when rapidly cooled through the order-disorder transition temperature. Following this process at lower temperature the crystal consists of ultrafine intergrowths of disordered (antiferromagnetic) and ordered (ferrimagnetic) phases, that is, the x-phase and the self-reversing phase, respectively. If the interface between these adjacent phases is coherent (Putnis, 1992), it is conceivable that exchange interactions can result in the magnetic moments of the two phases being forced to be antiparallel, although no specific mechanism was proposed by Uyeda. According to Uyeda's model, the two coexistent phases have the same chemical composition (i.e. the same value of $y$ ). However, this requirement appears to be at odds with production of reverse thermoremanent magnetization in the strongly ferromagnetic phase, since the Curie temperatures of synthetic hemoilmenite crystals of given composition are found to rise (not fall) with the degree of cation ordering (Ishikawa, 1958; Nord and Lawson, 1992). This experimental fact violates the perceived role of the $\mathrm{x}$-phase. Hence, this initial version of this model has been abandoned (Hoffman, 1975).

Another version of the two-phase model was proposed following the first examination of synthetic hemoilmenite samples by transmission electron microscopy (TEM) [Lawson et al. (1981); Nord and Lawson (1989, 1992]. These observations led these authors, and Hoffman (1992), to propose that the xphase is the boundary layer (APB) between adjacent cation antiphase (or, more exactly, twin) domains 
produced by a nucleation and growth cation ordering process. Hoffman (1992) argued that symmetry and lattice energy properties of hemoilmenite crystals require APBs to be both Fe-enriched and cationdisordered, giving rise to a weak spontaneous magnetization via a hematite-like spin-canted antiferromagnetic structure. According to Nord and Lawson (1992), twin boundaries consist of only a few atomic layers, and may pin down thin unconventional $180^{\circ}$ magnetic domain walls which cannot move. Thus, although multidomain (MD), the magnetic structure of crystals could exhibit high coercivity, as observed for both synthetic (Brown et al., 1993) and natural (Goguitchaivili and Prévot, 2000) hemoilmenites.

Hoffman (1992) examined the question of the exact mechanism of acquisition of self-reversed TRM. He pointed out two main problems with this proposed model. First, if the spontaneous magnetization js of the disordered phase is due to spin canting, then the spins of this phase-which would magnetically order first during cooling — should be perpendicular to the ambient magnetic field, not parallel to it. Since the exchange interaction is a spin-spin interaction (not a js-js interaction), it is unclear how the TRM carried by the cation ordered domains could then be self-reversed with respect to the ambient field. Second, such a spin-canted structure of the boundaries would produce opposite (antiparallel) directions of spontaneous magnetization in any two adjacent twin-domains. If so, then the net TRM of the assemblage of cation-ordered domains present in a single crystals should be zero-with no self-reversed component. To solve these major difficulties and at the same time explain thermomagnetic experimental findings on synthetic hemoilmenite crystals, Hoffman (1992) proposed a mechanism whereby below about $200^{\circ} \mathrm{C}$ the directions of spin within any two adjacent antiphase cation-ordered domains (i.e. separated by an APB) undergo symmetric rotations such that a reverse TRM component appears and grows with decreasing temperature. Although theoretically solving the problem, this hypothesis is difficult to confirm experimentally.

Model 2 (three-phase model). Alternatively, Ishikawa and Syono (1962) had proposed that the xphase is a Fe-enriched metastable phase that forms on the edge of the cation-ordered domains while each grows at the expense of disordered phase material. Provided that diffusion of metallic ions in these hexagonal crystals takes place more easily within basal plane layers than between adjacent cation layers (i.e. along the c-axis), they noted that the buffer region between each ordered and disordered domain is, in fact, the x-phase, that it would have to be partially ordered by the process, and that it would possess an antiphase cation relationship with the ordered phase. In other words, the mechanism of cation ordering would produce alternating Fe-enriched layers along the periphery that would align with alternating Fe-poor layers of the ordered phase, and vice-versa. Consequently, upon cooling the weakly ferrimagnetic magnetic moment of the $\mathrm{x}$-phase and that of the strongly ferrimagnetic ordered phase would be antiparallel, and selfreversal of TRM would result without calling for additional assumptions.

The above models have in common the hypothesis that the x-phase is Fe-enriched. Also, based upon theoretical considerations or experimental evidences (Ishikawa and Syono, 1963; Westcott-Lewis and Parry, 1971b; Hoffman, 1992), it is generally believed that reversed TRM can only be produced in grains having small cation-ordered domains, preferably of single domain (SD) size. Specifically, Nord and Lawson (1992) found that the TRM of large MD-sized hemoilmenite crystals with $y=0.7$ is reversed only when twin-domain sizes were smaller than $80 \mathrm{~nm}$; crystals with larger cation-ordered domains were found to carry a normal TRM.

Which one of these two models-both developed primarily from the study of synthetic hemoilmenite samples-is most applicable to volcanic rocks carrying a naturally self-reversed TRM is still a matter of debate. No decisive arguments for choosing between them have thus far been made from magnetic studies of self-reversed volcanic rocks (e.g. Nagata et al., 1952; Kennedy and Osborne, 1987; Lawson et al., 1987; Haag et al., 1990; Bina et al., 1999). Indeed, the study of natural hemoilmenite is more difficult, complicated in large part by the fact that this mineral, when found, has not been the only magnetic remanence carrier present, and interpretation of paleomagnetic and rock magnetic data has often been rather speculative. In addition, there are strong indications from TEM observations that the structure of cation-ordered domains in most synthetic hemoilmenite is quite distinct from naturally occurring samples. Specifically, synthetic minerals exhibiting self-reversal are typically characterized by a large volumetric dominance of cation-ordered domains with boundaries that are much thinner than their own dimensions (Nord and Lawson, 1992). In contrast, the only TEM observation so far reported for natural hemoilmenite (from Mt. Shasta, Lawson et al., 1987) reveals very small cation-ordered domains, possibly of SP size, embedded in an apparently disordered matrix comprising more than half of the total volume. 
Thus, a distinct self-reversal model may be needed for naturally occurring hemoilmenite (Goguitchaivili and Prévot, 2000).

In an attempt to constrain more precisely the self-reversal mechanism in natural hemoilmenite, we report here new mineralogical and magnetic data obtained from the self-reversed Pinatubo dacitic pumice. Our TEM and thermomagnetic observations lead us to propose a three-phase model, more specific than past attempts, which appears able to account for the characteristics of self-reversed TRM of the Pinatubo dacite and other similar volcanics.

\section{Review of previous work on the Pinatubo dacite}

The eruption of the Pinatubo dacitic pumice occurred on June 15, 1991. The temperature of the dacitic melt prior to eruption was estimated at $820 \pm 30^{\circ} \mathrm{C}$ at a depth of $8-10 \mathrm{~km}$ in the magmatic chamber (Rutherford, 1993; Hattori, 1993). Progressive oxidation of the magma has been claimed in order to explain the enrichment in $\mathrm{Fe}_{2} \mathrm{O}_{3}$ observed in the outer margins of some plagioclase phenocrysts (Hattori and Sato, 1996). This oxidation process is probably also the cause of core-to-margin chemical zoning found in some of the hemoilmenite crystals (Hoffmann and Fehr, 1996). Specifically, the cores of these grains, typically having a composition of $\mathrm{y} \cong 0.54$, are surrounded by slightly more Fe-enriched margins having a composition of $\mathrm{y} \cong 0.53$ (Bina et al., 1999). Hemoilmenite grains which are homogeneous at the microscopic level also are found in this lava; their chemical composition seems to be identical to that of the margins in zoned crystals (i.e. typically $\mathrm{y} \cong 0.53$ ). Note also that magma ascent of other dacitic melts has been found to be rapid; those associated with the Mount St. Helens 1980 eruptions have been reported to vary from a few hours to several tens of days (Rutherford, 1993).

Ozima et al. (1992) and Bina et al. (1999) showed that the natural TRM of the 1991 Pinatubo dacite is self-reversed. These authors and Hoffmann and Fehr (1996) observed the presence of two main magnetic phases, both appearing as macrocrystals typically a few hundreds of $\mu \mathrm{m}$ in size: titaniferous magnetite with $\mathrm{Tc} \cong 480-490^{\circ} \mathrm{C}$ and hemoilmenite with $\mathrm{Tc} \cong 250-280^{\circ} \mathrm{C}$, determined from the thermal variation of high or low-field induced magnetizations. All of these authors attribute the self-reversal of natural TRM solely to the hemoilmenite. This interpretation was verified by Goguitchaivili and Prévot (2000) who showed that a single crystal of hemoilmenite extracted from the Pinatubo dacite does indeed acquire a direction of TRM that opposes that of the laboratory applied field. These magnetic measurements on oriented single crystals also showed that the magnetic moment lies in the basal plane of the hexagonal structure, and that a triaxial anisotropy is present, presumably of magnetocrystalline origin.

Microscopic observations using ferrofluids (Hoffmann and Fehr, 1996; Bina et al., 1999) indicate that hemoilmenite crystals in the Pinatubo dacite present a MD magnetic structure. The slightly Fe-poorer cores of zoned crystals are strongly magnetic (SM), interpreted by these authors to be ferrimagnetic. In contrast, the slightly Fe-richer margins of zoned crystals and the homogeneous crystals are both weakly magnetic (WM), interpreted to be antiferromagnetic with a weak "parasitic" ferromagnetism due to spin canting. These observations led Hoffmann and Fehr (1996) to propose a MD two-phase model of selfreversal in which the self-reversed TRM is carried by the SM Fe-poorer regions as a result of some negative interaction acting solely at the interface with the surrounding slightly Fe-enriched WM crystal margin. This model is disputable for two main reasons. First, it is difficult to understand how APB-free cation-ordered regions reaching several tens of $\mu \mathrm{m}$ in size can be forced to carry a net TRM antiparallel to the applied field considering the very short range of superexchange interactions. Theoretical considerations suggest (Ishikawa and Syono, 1963; Westcott-Lewis and Parry, 1971b) and TEM observations confirm (Nord and Lawson, 1992) that cation-ordered domains have to be very finely subdivided by APB for a reversed TRM to be acquired. Second, as mentioned above, we found that the magnetic domain walls within the SM regions of the Pinatubo hemoilmenite macrocrystals can be easily moved by external fields smaller than 10mT. However, the reversed natural TRM of single crystals exhibits no soft reversed TRM. Basically, natural TRM is unaffected by alternating fields smaller than $40 \mathrm{mT}$ (Goguitaichvili and Prévot, 2000). Thus, the strongly magnetic regions of the zoned macrocrystals are negligible as TRM carriers. Given the fact that magnetic measurements suggest SD-like behavior of the cation-ordered regions in Pinatubo hemoilmenite, Goguitchaivili and Prévot (2000) proposed a different two-phase model in which the self-reversed TRM is carried by fine, cation-ordered domains dispersed in a matrix of cation-disordered slightly Fe-richer regions. In their model, like in the 3-phase model we propose below, no significant TRM is claimed to be carried by the magnetically soft Fe-poorer ferrimagnetic core of the zoned crystals. 


\section{New mineralogical and magnetic observations}

3.1. Magnetic Force Microscopy (MFM) observations.

MFM observations were carried out at Instrumat Company (Les Ulis) using Nanoscope 3a from Digital Instrument. In agreement with previous observations of the Pinatubo hemoilmenite using the Bitter technique (Hoffmann and Fehr, 1996; Bina et al., 1999), MFM imaging reveals a multidomain structure for both the strongly magnetic (Fig. 1a-c) and the weakly magnetic regions of zoned crystals (and homogeneous crystals) (Fig. 1e and f). A typical MD structure is also exhibited by titanomagnetite crystals (Fig. 1d).

Strongly magnetic regions are typically several tens of $\mu \mathrm{m}$ in diameter. The observed width of the magnetic domains varies between 0.5 and $5 \mu \mathrm{m}$. As pointed out above, the magnetic domain walls are easily moved by external fields of moderate magnitude. Thus, they are not somehow blocked by twin domain boundaries (APBs) as proposed by Nord and Lawson (1992) and Hoffman (1992) for synthetic equivalents. In contrast to the strongly magnetic regions, the weakly magnetic hemoilmenite regions (Fig. 1e and f) show quite large magnetic domains reaching several tens of $\mu \mathrm{m}$ in size and separated by irregularly undulating walls. Unlike the walls of the SM regions, these walls were found to be hard to move by external fields (Hoffmann and Fehr, 1996).

3.2. Transmission electron microscopy.

Dark-field transmission electron micrographs of hemoilmenite crystals were recorded in two beam conditions with the ordering reflection g=0003 using a Philips $300 \mathrm{kV}$ CM 30 model at Lille University. These micrographs show the presence of very fine cation-ordered domains in a cation-disordered matrix (Fig. 2). The dimensions of these cation-ordered domains vary between 20 to $40 \mathrm{~nm}$. Multiple layers of these "nano-domains" can be present in a given analyzed 100nm-thick section. Assuming a face-centered cubic distribution of the nano-domains, we estimated that the average distance between them is about $70 \mathrm{~nm}$. Assuming further that these cation-ordered domains are flattened in the basal plane (Lawson et al., 1981 ) as well as isometric within this plane, the average nano-domain volume is about $30 \times 30 \times 20 \mathrm{~nm}^{3}$. Using these values, the disordered matrix was found to represent approximately $95 \%$ of the total volume involved. The abundance of cation-disordered phase material observed in the sections studied by electron microscopy is consistent with the findings at the less-resolute microscopic level (Bitter technique, MFM and microprobe observations) which indicate a dominant phase that is overall weakly magnetic and slightly Fe-enriched $(\mathrm{y} \cong 0.53)$. That is, although homogeneous at the microscopic scale, these regions are in fact crystallographically heterogeneous. Moreover, these high-resolution observations suggest that much of the magnetization of these regions is not due to spin-canted antiferromagnetism, but rather evidently to the tiny ferrimagnetic cation-ordered domains. However, the magnitude of the spontaneous magnetization of these cation-ordered nano-domains remains unknown, since the degree of ordering cannot be determined from TEM analyses.

\subsection{Thermomagnetic analyses of TRMs}

Thermal demagnetization of remanence carried by rocks is easily interpreted in terms of unblocking temperatures when the magnetic phases present are not interacting. In the present case where as many as three mineralogical phases can magnetically interact with each other, special care is needed for interpreting thermomagnetic curves. The usual 'thermal demagnetization curve' obtained from measurements at room temperature after successive temperature cycling is particularly difficult to interpret. Less ambiguous information can be obtained from thermomagnetic curves obtained from magnetization measurements at temperature. Still, the interpretation of a decrease in remanence at some 'destructive temperature' $T_{d}$ is not straightforward. Its significance depends upon the relative values of the unblocking and Curie temperatures of the phases and the way they interact.

Fig. 3 shows the thermal demagnetization curves of natural and laboratory TRMs obtained from (a) a whole rock sample and (b) a single hemoilmenite crystal using an Orion thermomagnetometer. We note first that the demagnetization curves of laboratory weak-field TRMs obtained after an initial cooling from $600^{\circ} \mathrm{C}$ are similar for the single crystal and the rock sample. The only difference is that the latter exhibits a small normal component beyond $300^{\circ} \mathrm{C}$ which is not seen on the crystal curve. We attribute this component to titanomagnetite, and will not consider it further. Three main conclusions can be drawn from Fig. 3a, the whole rock results.

1. TRM consists of two components. The lower temperature component is self-reversed and largely dominant near room temperature. For the natural TRM, the destructive temperature $T_{d}$ of this component is about $270^{\circ} \mathrm{C}$. The higher temperature component is normal and $T_{d}$ is 
about $380^{\circ} \mathrm{C}$. This large temperature difference indicates that the normal component is carried (or blocked) by a phase much richer in iron than the carrier of the self-reversed component.

2. The normal TRM component is too large to be due to spin canting of an antiferromagnetic phase. Since the spontaneous magnetization of hemoilmenite varies linearly with temperature (Brown et al., 1993), the minimum magnitude of this component at room temperature can be roughly estimated from the decrease of the higher temperature segment of the curve on Fig. 3a. We find the magnitude of the room temperature normal TRM component to be at least 1/3 that of the self-reversed component. Thus, the carrier of the normal TRM must be ferrimagnetic, which implies some degree of cation-ordering.

3. The first heating to $600^{\circ} \mathrm{C}$ produces an irreversible change resulting in a translation of the demagnetization curve towards lower temperatures. The destructive temperatures of the normal and reversed components are lowered in concert, indicating a migration of iron ions from the cation-ordered phases. Considering the value of the peak temperature of heating, this change is probably due to spinodal decomposition (Fig.4), a process that has been observed in synthetic hemoilmenite within large cation-ordered domains (Lawson et al., 1981). However, spinodal decomposition should affect differently small cation-ordered domains whose size is only on the order or smaller than the spinodal wavelength (Allen and Cahn, 1976; Lawson, 1981), which corresponds to the present situation. In this case, it is expected that iron migrates from the small cation-ordered domains into the disordered phase while titanium move oppositely. Although some increase of the ordering degree can result from these migrations, which would tend to increase the Curie point, the overwhelming effect is the decrease in Curie temperature of the cation-ordered nano-domains due to the decrease in their $\mathrm{Fe} / \mathrm{Ti}$ ratio.

Experiments on laboratory TRMs indicate that destructive temperatures and those of magnetization acquisition are about the same. Figure 5 compares the acquisition and demagnetization of $\operatorname{TRM}\left(580^{\circ} \mathrm{C}, 50 \mu \mathrm{T}, \mathrm{T}_{0}\right)$ where $\mathrm{T}_{0}$ is room temperature. The acquisition curve lies above the demagnetization curve because of the additional magnetization induced by the field applied during cooling. Most of the offset is due to the increase in titanomagnetite susceptibility near the Curie temperature, found to average $470 \pm 12^{\circ} \mathrm{C}$ as measured by the inflection point (Prévot et al., 1983). As can be seen in Fig. 5, the acquisition and demagnetization curves would be very similar in the absence of the induced magnetization superimposed on the remanence cooling curve. The same conclusion was reached by Nagata et al. (1952) for the Mt. Haruna dacite.

\subsection{Interpretation and conclusions}

As pointed out by Goguitchaivili and Prévot (2000), the macro-sized strongly magnetic cores of the zoned hemoilmenite crystals seem fully cation-ordered and carry no more than very weak and softly coercive remanent components unrelated to the self-reversal process. Thus, we argue that the thermomagnetic data depicting highly coercive thermoremanence (Bina et al., 1999; Goguitchaivili and Prévot, 2000) provide information, not about the SM cation-ordered crystal cores, but rather about the nano-sized magnetic carriers observed by TEM in the weakly magnetic mostly cation-disordered regions. Since we are mainly interested in the mechanism of self-reversal as it occurs in nature, we consider below thermomagnetic data associated with natural TRM rather than laboratory TRM. Note also that all TEM experiments were carried out on unheated hemoilmenite crystals.

We claim that when combined the TEM and thermomagnetic observations indicate that three mineralogical phases comprise the weakly magnetic macrocrystals (or WM regions of zoned macrocrystals) of the Pinatubo hemoilmenite:

- a self-reversed cation-ordered phase, magnetically dominating the thermoremanence. Since selfreversed TRM is assumed to be blocked by a normally magnetized higher Curie temperature phase, the destructive temperature (about $270^{\circ} \mathrm{C}$ ) of this negative component should correspond to its Curie point. This value is in good agreement with the Curie temperatures reported by Ishikawa (1958) for ordered hemoilmenite with bulk compositions of $\mathrm{y}=0.51$ and 0.56 . This value corresponds also to the Curie temperature of hemoilmenite measured on whole rock Pinatubo samples (Ozima et al., 1992; Hoffmann and Fehr, 1996; Bina et al., 1999). Yet, careful weak-field thermomagnetic experiments on whole rock samples (Bina et al., 1999) failed to detect more than a single Curie point which can be attributed to hemoilmenite. This suggests that the tiny cation-ordered domains in the weakly magnetic regions and the large cation-ordered domains constituting the strongly magnetic core of some 
macrocrystals have about the same chemical composition ( $y \cong 0.54$, according to electron microprobe measurements).

- a normally magnetized partially cation-ordered phase, yielding also a quite conspicuous magnetic signal. As we will see in the following section, the magnetic moment of this phase is presumably blocked by a higher Curie point aureole. Thus, the destructive temperature of about $380^{\circ} \mathrm{C}$ can also be interpreted as a Curie temperature. According to Uyeda (1957), this temperature corresponds to a composition $\mathrm{y} \cong 0.35$.

- a cation-disordered phase, antiferromagnetic with presumed spin canting, which is the dominant phase seen in TEM (Fig. 2) in the weakly magnetic hemoilmenite macrocrystals and weakly magnetic regions of zoned macrocrystals. Electron microprobe data indicate that the bulk chemical composition of this phase corresponds approximately to $\mathrm{y} \cong 0.53$ (Bina et al., 1999). Due to the weakness of its spontaneous magnetization and insufficient sensitivity of our equipment, this phase was not observable during thermomagnetic runs. Thus, its Curie temperature (or Néel point) was unmeasurable. However, according to data from Ishikawa's (1958), a disordered phase with this composition should have a Curie point of about $200^{\circ} \mathrm{C}$. This indirectly determined value assumes that the embedded cation-ordered nano-domains have no pronounced effect on the chemical composition of the disordered matrix, a reasonable assumption given the TEM-observed volumetric importance of the disordered phase relative to the tiny ordered domains in the WM region. As will be seen, some local Fe-ion enrichment can be expected only along the contacts between these two phases.

\section{Self-reversal model}

For the reasons given above, we maintain that the cation-ordered, strongly ferrimagnetic slightly Fe-poor core of the zoned Pinatubo hemoilmenite plays no role in the self-reversal process. Rather-and central to the new self-reversal model which we now develop-we claim that only material comprising the weakly magnetic hemoilmenite crystals, and WM crystal margins of zoned crystals, is involved in the process.

\subsection{Sequence of mineralogical transformations during magma cooling}

Hemoilmenite and titanomagnetite are commonly the first phases to crystallize from a dacitic melt, typically at temperatures of about $910 \pm 30^{\circ} \mathrm{C}$ (Lawson et al., 1987). After an apparently long storage time in the magma chamber at approximately $820^{\circ} \mathrm{C}$, magma cooling is probably negligible during ascent (see Rutherford, 1993). Significant cooling occurrs rapidly at first during the short time between volcanic explosion and deposition (to temperatures probably exceeding $460^{\circ} \mathrm{C}$ according to Bina et al., 1999), and then quite slowly.

As shown in the temperature-composition phase diagram (Fig. 4), two mineralogical transformations can occur successively during cooling, namely, cation-ordering (below $700-750^{\circ} \mathrm{C}$ ) and spinodal decomposition (below about $650-700^{\circ} \mathrm{C}$ according to Lawson; 1981). Ishikawa and Syono (1963) have shown that the critical temperature of the order-disorder transformation in this solid solution series dramatically increases with ilmenite content. The observations reported here for the Pinatubo hemoilmenite suggest that the quenching temperature of the dacitic magma, estimated to be in the range $790-850^{\circ} \mathrm{C}$, straddles the order-disorder critical temperatures for compositions between $\mathrm{y}=0.53$ and $\mathrm{y}=$ 0.54 . On the one hand, the $y=0.54$ material, being held below its order-disorder critical temperature within the magma chamber, would have possessed a small, yet non-zero, homogeneous equilibrium degree of cation order at that temperature well before the eruption. This initial ordered state would facilitate rapid ordering during the quench, producing a far higher (perhaps, nearing equilibrium) degree of order at low temperature. On the other hand, the $\mathrm{y}=0.53$ material, being held above its order-disorder critical temperature within the magma chamber, would have been disordered at the time of the quench. Cation ordering during the quench is then likely to be confined to tiny regions possessing at that time a slightly higher Ti-ion concentration. As discussed in the previous sections, magnetic ordering occurs later at temperatures between about 400 (disordered Fe-enriched aureole) and $200^{\circ} \mathrm{C}$ (disordered matrix).

\subsection{Mineralogical model}

Our key assumption is that the ferrimagnetic phase carrying the normal TRM component seen on the thermomagnetic curves corresponds to the x-phase of Ishikawa and Syono $(1962,1963)$. According to this conception, the x-phase is a partially ordered buffer zone located between disordered material and fully ordered domains. Due to a slightly richer Fe content the buffer zone magnetizes first, acquiring a net normal magnetic moment. Upon further cooling, this moment is opposed by a far more intense moment growing within the ordered domain. This stronger moment is reversed because of the superexchange 
interactions and the cation antiphase nature of the fully ordered material relative to the adjacent buffer zone.

These simple and general considerations are in principle sufficient to explain self-reversal. However, they have to be reconciled with the apparently contradictory observations that the cation-ordered nano-domains are presumably of superparamagnetic size while they carry a normal remanence which is not destroyed before reaching a temperature of $380^{\circ} \mathrm{C}$, largely in excess of the estimated Curie/Néel temperature of the disordered matrix $\left(200^{\circ} \mathrm{C}\right)$ surrounding these domains. It is clear that, at room temperature, the x-phase cannot behave "alone", as a SP particle, because it is surrounded by the disordered matrix which blocks the spins of the nano-domains at their boundary through superexchange interaction. However, beyond $200^{\circ} \mathrm{C}$, the cation-ordered domains are dispersed particles in a paramagnetic medium. Thus, if they are of SP-size, they should then loose their remanence.

According to TEM observations, and in the absence of interactions with another phase, the $x$ phase should in fact be superparamagnetic at and above room temperature. Although only partially ordered, this phase is expected to yield a $\mathrm{g}=0003$ reflection under the electron beam. Thus, the $\mathrm{x}$ phase should constitute only part of the tiny 20-40 nm cation-ordered domains seen with TEM. Given its estimated molecular ilmenite fraction ( $\mathrm{\cong} 0.35$ ), it is doubtful that the $\mathrm{x}$-phase can have unblocking temperatures as high as the destructive temperature of about $380^{\circ} \mathrm{C}$ observed for the normal TRM component. The calculated superparamagnetic threshold size $d_{s}$ for SD hemoilmenite at room temperature is $35 \mathrm{~nm}$ for $\mathrm{y}=0.60$ and $100 \mathrm{~nm}$ for $\mathrm{y}=0.50$ according to Lawson (1981), and a value of $25-30 \mathrm{~nm}$ is commonly reported for hematite

In order to solve this contradiction, our second main assumption is that the magnetic moments of the $\mathrm{x}$-phase are blocked by exchange interactions with the surrounding disordered phase. In order to lock the spins of the $\mathrm{x}$-phase up to, say, $400^{\circ} \mathrm{C}$, the disordered phase in the vicinity of the cation-ordered domains must have a Curie temperature at least equal to this temperature. This requires some local ironenrichment of the disordered matrix during magma cooling. Both cation ordering and spinodal decomposition are expected to produce such a local Fe-enrichment of the disordered matrix at the expense of the cation-ordered domains (Allen and Cahn, 1976; Lawson, 1981; Lawson et al., 1981; Nord et Lawson, 1989; Hoffman, 1992). The mineralogical model we propose (Fig. 6) assumes, therefore, that beyond the buffer zone (x-phase) constituting the outer part of the cation-ordered domains seen in TEM lies an external aureole of Fe-enriched disordered material. Note that superexchange interactions must act on both the inner and outer interfaces of the partly ordered x-phase. The spins of this phase are thus locked to those of the adjacent atomic layers of both the surrounding disordered aureole and the fully cation-ordered core of the tiny domain.

\subsection{Acquisition of self-reversed TRM}

As already noted, thermomagnetic measurements at temperature do not provide information about the unblocking range of the Fe-enriched aureoles, which are presumably of single domain size. However, assuming that our interaction model is valid and following Hoffman (1975), this information can be retrieved from stepwise thermal demagnetization of the self-reversed NRM measured at room temperature $\mathrm{T}_{0}$. The demagnetization curve of a single hemoilmenite crystal heated in air (Goguitchaivili and Prévot, 2000) starts with a perfect plateau continuing up to $330^{\circ} \mathrm{C}$, a temperature that significantly exceeds the destructive temperature of the self-reversed phase. We interpret this stability by the effect of magnetic interactions acting successively during cooling in zero field. First the x-phase and then the self-reversed phase will remagnetize in an identical way during each cooling run so long as the range of unblocking temperatures of the higher Curie point Fe-enriched aureoles - the spins of which determine through superexchange the spin direction and sense of the x-phase-has not been reached. Thus the unblocking range of $\operatorname{NRM}\left(T, T_{0}\right)$ can be interpreted as the unblocking range of Fe-enriched aureoles. Mineralogical changes are probably responsible for the tail of the $\operatorname{NRM}\left(T, T_{0}\right)$ curve at higher temperature observed by Goguitchaivili and Prévot (2000). Thus, we estimate that the unblocking range of $\operatorname{NRM}\left(T, T_{0}\right)$ extends from $330^{\circ} \mathrm{C}$ to about $410^{\circ} \mathrm{C}$. That is, the maximum unblocking temperature of Fe-enriched aureoles is about $30^{\circ} \mathrm{C}$ higher than the destructive temperature of the normal component of natural TRM. Considering $410^{\circ} \mathrm{C}$ to be a minimum estimate of the Curie point of these cation-disordered Fe-enriched aureoles, their composition is $\mathrm{y}<0.3$.

Figure 7 describes the proposed model of reverse-TRM acquisition assuming that the field direction during cooling coincides with an easy axis of magnetization in the basal plane. As temperature falls below $410^{\circ} \mathrm{C}$, the Fe-enriched disordered aureoles magnetize progressively. Assuming a spin-canted antiferromagnetic structure, the magnetic moments are weak and oriented at right angle to the spins. Due to the triaxial anisotropy in the basal plane, the magnetic moments are directed at $\pm 30^{\circ}$ from the ambient field 
direction and the spins lie at $\pm 60^{\circ}$ from the field (Fig. 7b). Below $380^{\circ} \mathrm{C}$, the partially ordered $\mathrm{x}$-phase becomes magnetized with a much larger moment which is parallel to the spins. In this figure, we make the assumption (discussed below) that the magnetic torque due to the ambient field acting on this moment, combined with the spin-to-spin interaction and the weak anisotropy in the basal plane, forces the spins of the whole system (aureole $+x$-phase) to rotate by $60^{\circ}$ in order to align the moment of the $x$-phase along the easy-axis direction of magnetization which is the closest to the applied field (Fig. 7c). Henceforth, the magnetic moment of the disordered matrix lies at right angles to the field direction. As temperature decreases below $270^{\circ} \mathrm{C}$, the fully ordered core of the tiny domains becomes magnetized. Since this phase is antiphase in terms of cation composition to the x-phase, the interlayer antiferromagnetic and intralayer ferromagnetic interactions cause the net magnetic moment to orient opposite to the field direction (Fig. 7d). Even if the fully cation-ordered phase and the x-phase are comparable in volume, the reversed magnetic moment of the ordered core should easily overwhelm the normal magnetic moment of the partially ordered x-phase as cooling proceeds. According to Wescott-Lewis and Parry (1971a), the saturation magnetization at room temperature is fifty times less for $y=0.35$ ( $y$ value of the $x$-phase) than for $y=0.57$ ( $y$ value of the self-reversed phase is 0.54 ). The last stage of TRM acquisition is the magnetic ordering of the whole matrix around $200^{\circ} \mathrm{C}$. Two cases have to be considered. If, on the one hand, the two nearest cation-ordered domains are compositionally in phase, so are the two disordered regions surrounding them. Thus, the entire disordered region between these ordered domains will coherently magnetize during cooling. The spins within each cation layer are parallel to each other and the weak magnetic moment of the disordered region, perpendicular to the field direction, will have the same sense everywhere. If, on the other hand, the cationordered cores are compositionally antiphase, then a magnetic domain wall must appear within the disordered matrix (Fig. 7e); otherwise the moment of one of the two types of antiphase domain would be forced to reverse and the thermoremanence of the whole macrocrystal would become zero below $200^{\circ} \mathrm{C}$. On both sides of this magnetic wall, magnetic moments of the disordered matrix material are perpendicular to the field direction, and possibly opposite to each other. Note that these walls are only of magnetic origin, and do not correspond to a crystallographic antiphase boundary. In 3-dimensional space, the probability for a given cation-disordered region to be surrounded only by antiphase domains is negligible, and it can be expected that domains in phase with one another tend to be interconnected (Allen and Cahn, 1976).

We wish to point out that our model predict the acquisition of a perfectly self-reversed TRM by a whole rock sample, independently of the validity of our hypothesis that the spins rotate by $60^{\circ}$ around $380^{\circ} \mathrm{C}$ (Fig. $7 \mathrm{~b}$ and c). In any case, the directions of the ferrimagnetic moments of macrocrystals are biased by the applied field and their net moment is parallel, or antiparallel, to the field direction provided the macrocrystal orientations are isotropic. In contrast, for a single oriented macrocrystal, one should observe that the direction of rTRM lie along the easy axis direction which is the closest to the applied field direction if the proposed spin rotation is real while this remanence should lie $60^{\circ}$ away from that direction in the absence of such a rotation. Goguitchaichvili and Prévot (2000) showed that partial self-reversed TRMs acquired in adjacent temperature intervals by a single oriented Pinatubo macrocrystal lie generally along the easy axis direction which is the closest to the applied field direction, with only 3 pTRMs out of 8 lying at $\pm 60^{\circ}$ away from that direction. These experimental results justify the $60^{\circ}$ rotation of the spins of the cation-disordered Fe-enriched aureole and adjacent x-phase proposed in our model.

\section{Discussion and conclusions}

Incipient cation-ordering was first observed in synthetic hemoilmenite. Nord and Lawson (1989) found very fine cation-ordered domains on order of $10 \mathrm{~nm}$ dispersed into disordered matrix in a synthetic hemoilmenite with $\mathrm{y}=0.60$ quenched from $900^{\circ} \mathrm{C}$. Even during extremely short quenching times (a few seconds) from very high temperature $\left(1300^{\circ} \mathrm{C}\right)$, magnetic measurements on synthetics with y compositions varying from 0.2 to 0.6 demonstrate that some incipient cation ordering occurs systematically (Brown et al., 1993). Similar incipient cation-ordering can be expected to occur in natural hemoilmenite crystals, depending upon the y value and the quenching temperature, which is estimated to vary approximately between 800 and $900^{\circ} \mathrm{C}$ for dacitic magmas (Rutherford, 1993). The comparison of this temperature interval with the lower limit of the disordered domain shown on the phase diagram (Fig. 4) suggests that hemoilmenite with y up to approximately 0.63 can lie above their order-disorder critical temperature within a dacitic magma chamber, and therefore be fully disordered at the time of the quench. Incipient cation order is then likely to occur during the quench, as described above for the $y=0.53$ regions of the Pinatubo hemoilmenite macrocrystals, and a reversed TRM can be acquired following our model. In agreement with 
these considerations, the self-reversing hemoilmenite found in dacitic pumice from mounts Haruna (Nagata et al.,1953), Natib (Kennedy and Osborne, 1987), Shasta (Lawson et al.,1987), and Pinatubo (Bina et al., 1999), contain a molecular fraction of ilmenite, $y$, that generally falls in the $0.53-0.56$ range, while in the andesitic dacite pumice from the 1985 eruption of Nevado del Ruiz y reaches $0.62 \pm 0.02$ (Haag et al., 1990). This range of variation of $y$ is included within the range for which incipient ordering is expected to occur under the natural temperature conditions. Indeed, the two self-reversing hemoilmenites from dacitic eruptions (Mount Shasta and White River) examined by Nord and Lawson (1989) in the TEM does show tiny cation-ordered domains similar to those observed in the Pinatubo crystals.

The ferrimagnetic nature of the x-phase proposed by Ishikawa and Syono $(1962,1963)$ has been demonstrated here to be valid for the Pinatubo self-reversing hemoilmenite. We believe that this conclusion may have general significance: the crucial experiment for testing this possibility consists of measuring the total TRM continuously in zero field while the temperature is progressively increased. To our knowledge, this kind of experiment only has been previously carried out on two self-reversed pumices, the Mt. Haruna dacite (Nagata et al., 1952) and the 1985 dacitic andesite pumice of Nevado del Ruiz (Heller et al., 1986). In both cases the result is unambiguous: the normal TRM component carried by hemoilmenite is much too large to be due to spin-canting. Somewhat similar experiments were carried out by Hoffman (1992) on two synthetic samples of self-reversing hemoilmenite with $y=0.6$ in which cation order was induced by way of different thermal treatments. The measurements were done under a normally directed $10 \mathrm{mT}$ field and relative to a rather high field TRM $(10 \mathrm{mT})$. The two samples behave differently. While one sample (slowly cooled from above the order-disorder transition temperature) exhibits a quite distinct positive hump, the other sample (annealed for over a week at $550^{\circ} \mathrm{C}$, that is, below the order-disorder transition temperature) shows the presence of only a very small normal TRM. Hoffman (1992) argues that the difference in these thermomagnetic behaviors arises from a differing size range of ordered, self-reversing regions produced by the thermal treatments (large MD and small SD regions the furnace-cooled and annealed samples, respectively). However, another possibility is that the latter thermal treatment (i.e. annealed sample) corresponds to a sample in which the total volume of $x$-phase produced is extremely small. Or, perhaps, certain synthetically produced hemoilmenites possess distinct properties from their natural counterparts. In any case, to our knowledge no other thermomagnetic studies of this kind have been carried out on synthetic material. Clearly, the validity of any 2-phase model as the explanation of the self-reversal of TRM in synthetic hemoilmenite has yet to be thoroughly proven.

A major difficulty with the 2-phase model of self-reversal is that the x-phase is supposed to correspond to APB-regions which, according to the TEM observations of Nord and Lawson (1992), are atomically thin, probably only a few angströms in thickness. If so, it seems difficult for this thin "ribbon" to have blocking temperatures large enough for exceeding those of the self-reversed phase, as required for self-reversing. Recent experiments on $\mathrm{Fe}_{3} \mathrm{O}_{4} / \mathrm{CoO}$ multilayers (van der $\mathrm{Zaag}$ et al., 2000) showed that the blocking temperature of the antiferromagnetic $\mathrm{CoO}$ layers starts to decrease rapidly towards $0 \mathrm{~K}$ as the layer thickness diminishes below $9 \mathrm{~nm}$. So, for a layer 5-angström thick, the blocking temperature is reduced to $20 \mathrm{~K}$. In contrast, a blocking temperature reaching almost the Néel temperature (291 K) characterizes $\mathrm{CoO}$ layers with thickness exceeding $9 \mathrm{~nm}$. Our 3-phase model of self-reversal is less demanding since it postulates the existence of crystallographic domains whose size is of the order of tens of nanometers. It is interesting to note that the size of cation-ordered domains observed by TEM in selfreversing synthetic or natural hemoilmenites varies rather largely, from 10nm (Larson and Lawson, 1989) to 100nm (Lawson et al., 1987). In our 3-phase model the crucial parameter is the size of the cationdisordered Fe-enriched aureoles which stabilize the spins of the x-phase and, thus, indirectly, the selfreversed magnetic moment. In the Pinatubo hemoilmenite, these aureoles correspond to an ilmenite fraction y less than 0.3. This high hematite content makes it reasonable to use the better known end-member (hematite) for a crude estimate of the volume needed for the magnetic moment of these aureoles to remain blocked up to $400^{\circ} \mathrm{C}$. Taking for hematite $d_{s}=30 \mathrm{~nm}$, we find that an aureole $15 \mathrm{~nm}$ wide is sufficient to stabilize the remanence of the cation-ordered nucleus up to this temperature. Although thin, this layer also seems to be of sufficient size for its Curie point not to be affected by nearby adjacent phases. Consistent with this argument are the recent experiments on $\mathrm{Fe}_{3} \mathrm{O}_{4} / \mathrm{CoO}$ multilayers (van der Zaag et al., 2000) which indicate that the Néel temperature of the antiferromagnetic $\mathrm{CoO}$ layers is unaltered provided they have a thickness of at least $10 \mathrm{~nm}$.

Although the present 3-phase model appears to be more compatible with the body of experimental data now available than previously proposed models, it remains, like them, a purely qualitative attempt to explain the enigma of self-reversal. Progress in micromagnetic computation of nanoparticles (Kachkachi et 
al., 2000; Kachkachi and Garanin, in the press) suggest, however, that it will soon become possible to check the physical robustness of such models.

\section{Acknowledgements}

We thank Subir Banerjee, Friedrich Heller and an anonymous reviewer for their useful comments about the first version of this paper. We also thank Anne Delplanque for her decisive help with drawing. This work was supported by CNRS-INSU project 'Terre intérieure'. A. Goguitchaivili was supported by CONACYT project J32727.

\section{References}

Allen, S. M., Cahn, J. W., 1976. Mechanisms of phase transformations within the miscibility gap of Fe-rich Fe-Al alloys. Acta metallurg., 24, 425-437.

Bina, M., Tanguy, J.C, Hoffmann, V., Prévot, M., Listanco, E.L., Keller, R., Fehr, K.Th., Goguitchaichvili, A.T., Punongbayan, R.S., 1999. A detailed magnetic and mineralogical study of self-reversed dacitic pumices from the 1991 Pinatubo eruption (Philippines), Geophys. J. Int., 138, 159-178.

Brown, N.E., Navrotsky, A., Nord, G.L.,. Banerjee, S.K, 1993. Hematite-Ilmenite $\left(\mathrm{Fe}_{2} \mathrm{O}_{3}-\mathrm{FeTiO}_{3}\right)$ solid solutions: Determination of Fe-Ti order from magnetic properties. Amer. Mineral., 78, 941-951.

Burton, B. P., 1991. The interplay of chemical and magnetic ordering. In Reviews in mineralogy, 25, 303321.

Goguitchaichvili A., 1999. Fluctuations du champ magnétique terrestre au Mio-Pliocène et auto-inversion de l'aimantation thermorémanente, Ph. D., Université de Montpellier 2, pp.271.

Goguitchaichvili A., Prévot M., 2000. Magnetism of oriented single crystals of hemoilmenite with selfreversed thermoremanent magnetization. J. Geophys. Res., 105, 2761-2780.

Haag, M., Heller F., Carracedo J. C., Soler V., 1990. Remanent magnetization of andesitic and dacitic pumice from the 1985 eruption of Nevado del Ruiz (Colombia) reversed due to self-reversal. J. Volc. Geoth. Res., 41, 369-377.

Hattori, K., 1993. High-sulfur magma, a product of fluid discharge from underlying mafic magma: evidence from ount Pinatubo, Philippines. Geology, 21, 1083-1086.

Hattori, K., Sato, H., 1996. Magma evolution recorded in plagioclase zoning in 1991 Pinatubo eruption products. Amer. Mineral., 81, 982-994.

Heller, F., Carracedo, J. C., Soler V., 1986. Reversed magnetization in pyroclastics from the 1985 eruption of Nevado del Ruiz, Colombia. Nature, 324, 241-242.

Hoffman, K. A., 1975. Cation diffusion processes and self-reversal of thermoremanent magnetization in the ilmenite-haematite solid solution series. Geophys.J.R. astr. Soc., 41, 65-80.

Hoffman, K. A., 1992. Self-reversal of thermoremanent magnetization in the ilmenite-hematite system: Order-disorder, symmetry, and spin alignment. J. Geophys. Res., 97, 10883-10895.

Hoffmann, V., Fehr, K. Th., 1996. Micromagnetic, rockmagnetic and mineralogical studies on dacitic pumice from the Pinatubo eruption (1991, Philippines) showing self- reversed TRM. Geophys. Res. Lett., 23, 2835-2838.

Ishikawa, Y., 1958. An order-disorder transformation phenomenon in the $\mathrm{FeTiO}_{3}-\mathrm{Fe}_{2} \mathrm{O}_{3}$ solid solution series. J. Phys. Soc. Japan, 13, 828-837.

Ishikawa, Y., Syono, Y., 1962. Reverse thermoremanent magnetism in the $\mathrm{FeTiO}_{3}-\mathrm{Fe}_{2} \mathrm{O}_{3}$ system. J. Phys. Soc. Japan, 17, suppl. B-I, 714-718.

Ishikawa, Y., Syono, Y., 1963. Order-disorder transformation and reverse thermo-remanent magnetism in the $\mathrm{FeTiO}_{3}-\mathrm{Fe}_{2} \mathrm{O}_{3}$ system. J. Phys. Chem. Solids, 24, 517-528.

Kachkachi, H., Ezzir, A., Noguès, M., Tronc, E., 2000. Surface effects in nanoparticles: application to maghemite $\gamma-\mathrm{Fe}_{2} \mathrm{O}_{3}$. Eur. Phys. J. B 14, 681-689.

Kachkachi, H., Garanin, D. A. Boundary and finite-size effects in small magnetic systems. J. Phys. Cond. Matter, in the press.

Kennedy, L. P., Osborne, M. D., 1987. Composite titanomagnetite-ferrian ilmenite grains and correlative magnetic components in a dacite with self-reversed TRM. Earth Plan. Sci. Lett., 84, 479-486.

Lawson, C. A., 1981. Magnetic and structural properties of minerals of the ilmenite-hematite solid solution series with special reference to the phenomenon of reverse thermoremanent magnetism. Ph. D., Princeton Univ., pp. 345. 
Lawson, C.A., Nord, G.L., Champion, D.E., 1987. Fe-Ti oxide mineralogy and origin of normal and reverse remanent magnetization in dacitic pumice blocks from Mt. Shasta, California. Phys. Earth Planet. Inter., 46, 270-288.

Lawson, C.A., Nord, G.L. Dowty, E., Hargraves R.B., 1981. Antiphase domains and reverse thermoremanent magnetism in ilmenite-hematite minerals. Science, 213, 1372-1374.

Nagata, T., Uyeda, S., Akimoto, S., 1952. Self-reversal of thermo-remanent magnetism in igneous rocks. J. Geomag. Geoelectr., 4, 22-38.

Nagata, T., Uyeda, S., Akimoto, S., 1953. Self-reversal of thermo-remanent magnetism in igneous rocks (III). J. Geomag. Geoelectr., 5, 168-184.

Néel, L., 1951. L’inversion de l'aimantation permanente des roches. Ann. Géophys., 7, 90-102.

Nord, G.L., Lawson, C.A., 1989. Order-disorder transition-induced twin domains and magnetic properties in ilmenite-hematite. Am. Mineral., 74, 160-176.

Nord, G.L., Lawson, C.A., 1992. Magnetic properties of ilmenite ${ }_{70}$-hematite $_{30}$ : effect of transformationinduced twin-boundaries. J. Geophys. Res., 97, 10897-10910.

O’Reilly, W., 1984. Rock and mineral magnetism. Blackie \& Son Ltd, Glasgow, 220 pp.

Ozima, M., Funaki, M. Hamada, N. Aramaki S., Fujii, T., 1992. Self-reversal of thermo-remanent magnetization in pyroclastics from the 1991 Eruption of Mt. Pinatubo, Philippines. J. Geomag. Geoelect., 44, 979-984.

Pokhil, T. G., Moskowitz, B. M., 1997. Magnetic domains and domain walls in pseudo-single-domain magnetite studied with magnetic force microscopy. J. Geophys. Res., 102, 22681-22694.

Prévot, M., Mankinen, E. A., Grommé, S., 1983. High paleointensities of the geomagnetic field from thermomagnetic studies on Rift Valley pillow basalts from the Mid-Atlantic ridge. J. Geophys. Res., 88, 2316-2326.

Putnis, A., 1992. Introduction to mineral sciences, Cambridge Univ. Press, 457 pp.

Rutherford, M. J., 1993. Experimental petrology applied to volcanic processes. EOS, Transactions, Amer. Geophys. Union, 74, 49-50.

Uyeda, S., 1957. Thermo-remanent magnetism and coercive force of the ilmenite-hematite series. J. Geomag. Geoelectr., 9, 61-78.

Uyeda, S., 1958. Thermo-remanent magnetism as a medium of paleomagnetism, with special reference to reverse thermoremanent magnetism. Jap. J. Geophys., 2 , 1-123.

Van der Zaag, P.J., L.F. Feiner, R.M. Wolf, J.A. Borchers, Y. Iriji, R.W. Erwin, 2000. The blocking and Néel temperature in exchange-biased $\mathrm{Fe}_{3} \mathrm{O}_{4} / \mathrm{CoO}$ multilayers. Physica B 276-278, 638-639.

Westcott-Lewis, M.F., Parry, L. G., 1971a. Magnetism in rhombohedral iron-titanium oxides. Austr. J. Phys., 24, 719-734.

Westcott-Lewis, M.F., Parry, L. G., 1971b. Thermoremanence in synthetic rhombohedral iron-titanium oxides. Austr. J. Phys., 24, 735-742. 


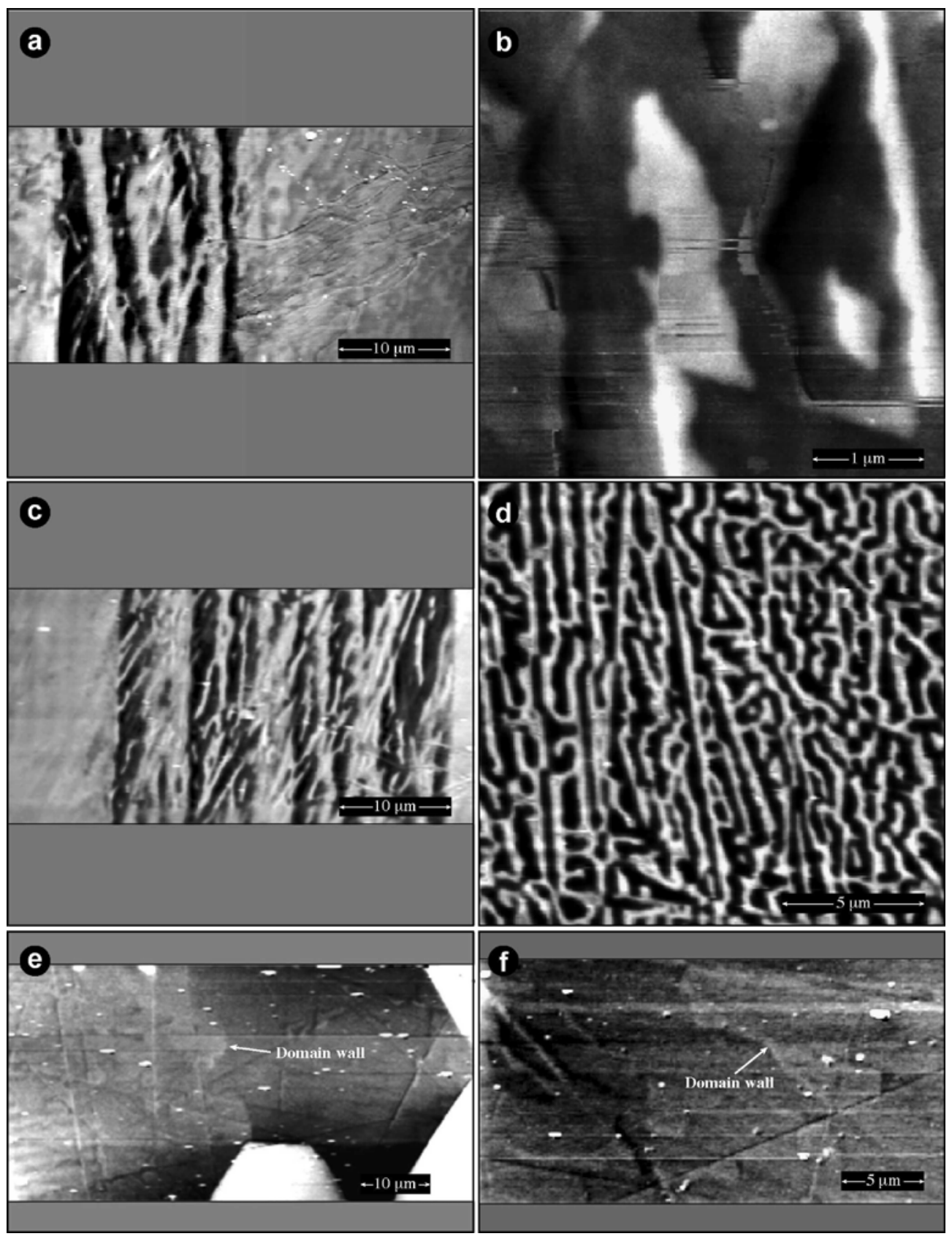

Figure 1. Magnetic Force Microscopy observations ( $a-c, e-f)$ of hemoilmenite crystals and a titanomagnetite crystal (d) from Pinatubo rock sample 9534 (Bina et al., 1999). Chemically zoned crystals $(a, b, c)$ are illustrated by two examples ( $a$ and $c$ ) showing a well developed, strongly magnetic core with magnetic domains of alternate polarity (light grey/black) flanked by a weakly magnetic crystal margin (medium grey) in which no domain walls are visible. (b) shows a detail of the SM region of the crystal shown in (a). In chemically homogeneous and weakly magnetic hemoilmenite crystals ( $e$ and $f$ ), magnetic domains are quite large and separated by faint irregular domain walls. White areas in $e$ correspond to silicate. In $(b, e, f)$ horizontal regular stripes are experimental artifacts parallel to the scanning direction during measurement. The micromagnetic structure of Ti-poor titanomagnetite $(d)$ is typical of this mineral (Pokhil and Moskowitz, 1997). 


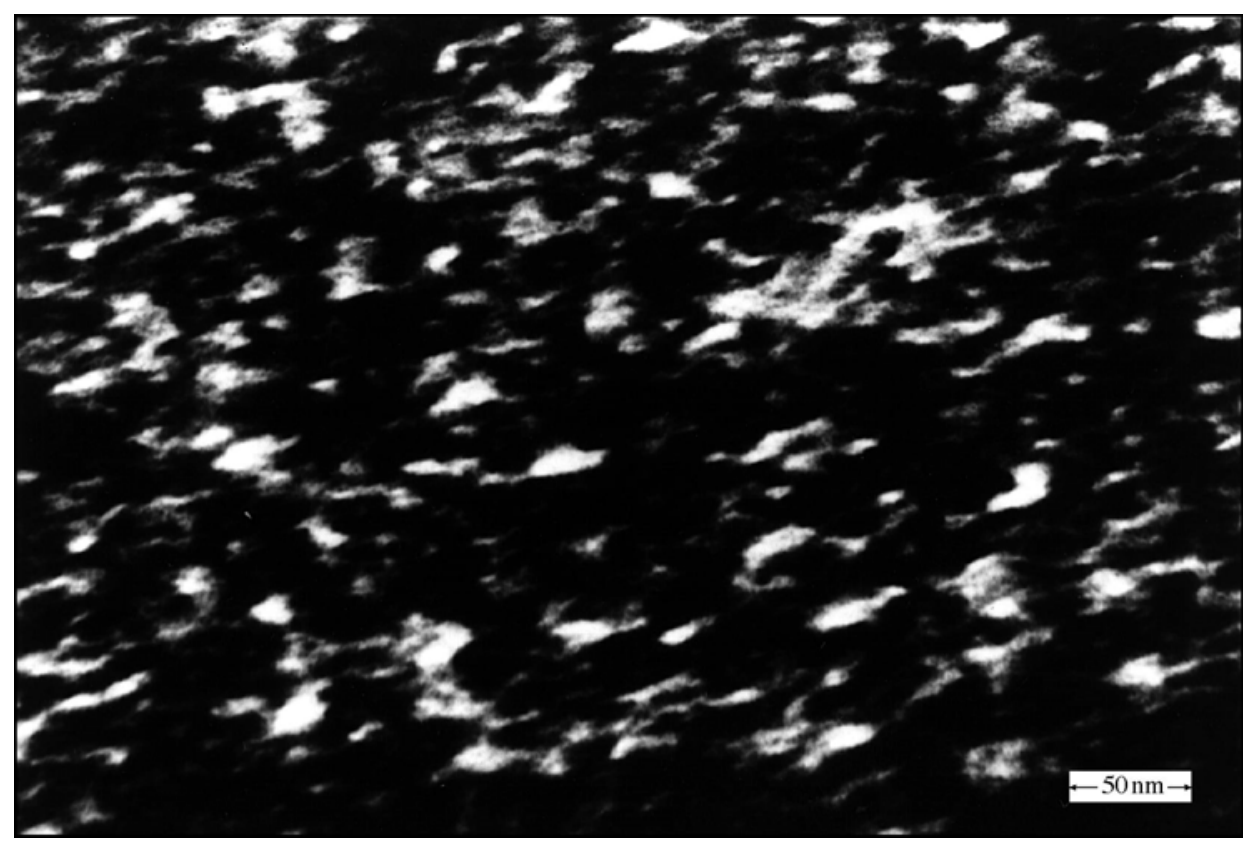

Figure 2. Dark-field transmission electron micrographs of a hemoilmenite crystal (weakly magnetic region) taken with the ordering reflection $\mathrm{g}=0003$ and showing tiny cation-ordered domains (typically 20-40nm)

in a cation-disordered matrix. Ordered domains tend to be flattened along the direction of the basal hexagonal plane.
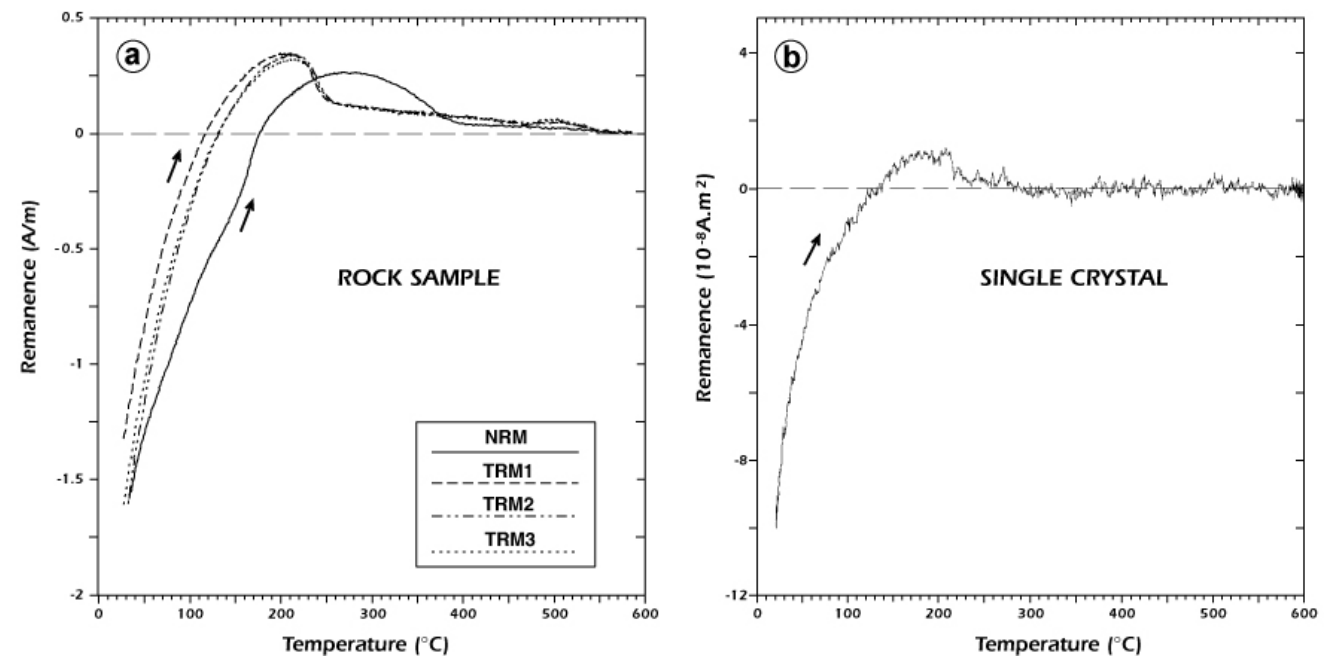

Figure 3. Continuous thermal demagnetization in zero field of total TRMs acquired by ( $a$ ) a whole rock sample (933-1, Bina et al., 1999), and (b) a single hemoilmenite crystal (400-36, Goguitchaichvili and Prévot, 2000) measured with an Orion VSTM (vibrating sample thermomagnetometer). In (a) the demagnetization curves shown correspond to the natural TRM (NRM) and 3 successive laboratory TRMs acquired from $600^{\circ} \mathrm{C}$ to room temperature in a $50 \mu \mathrm{T}$ field. (b) shows the demagnetization curve of a laboratory TRM acquired in the same temperature interval under a $0.3 \mathrm{mT}$ field. The only difference between whole rock and single crystal laboratory TRMs is the small normal component carried by titanomagnetite, which is the main magnetic phase in whole rock samples (Bina et al., 1999) 


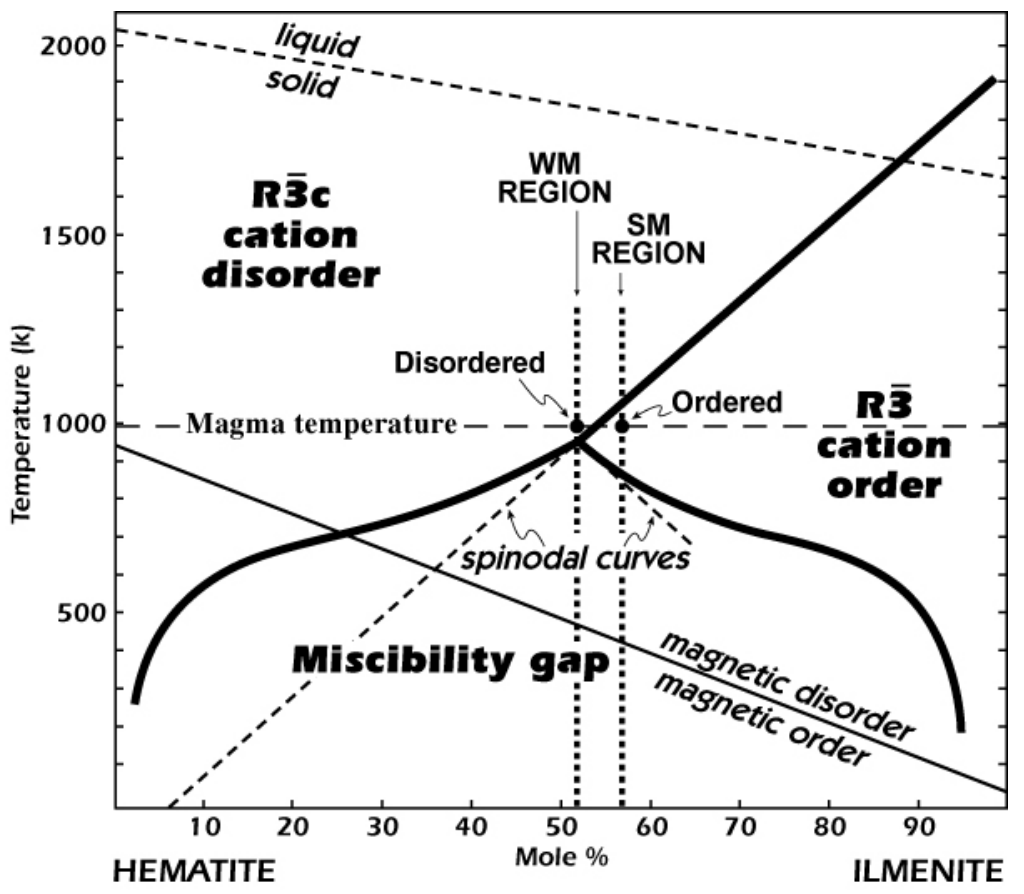

Figure 4. Phase diagram for the system hematite-ilmenite modified from Lawson (1981), Burton (1991) and Putnis (1992). To make the figure clearer, the chemical difference between weakly magnetic (WM) and strongly magnetic (SM) regions of Pinatubo hemoilmenite macrocrystals has been exaggerated.

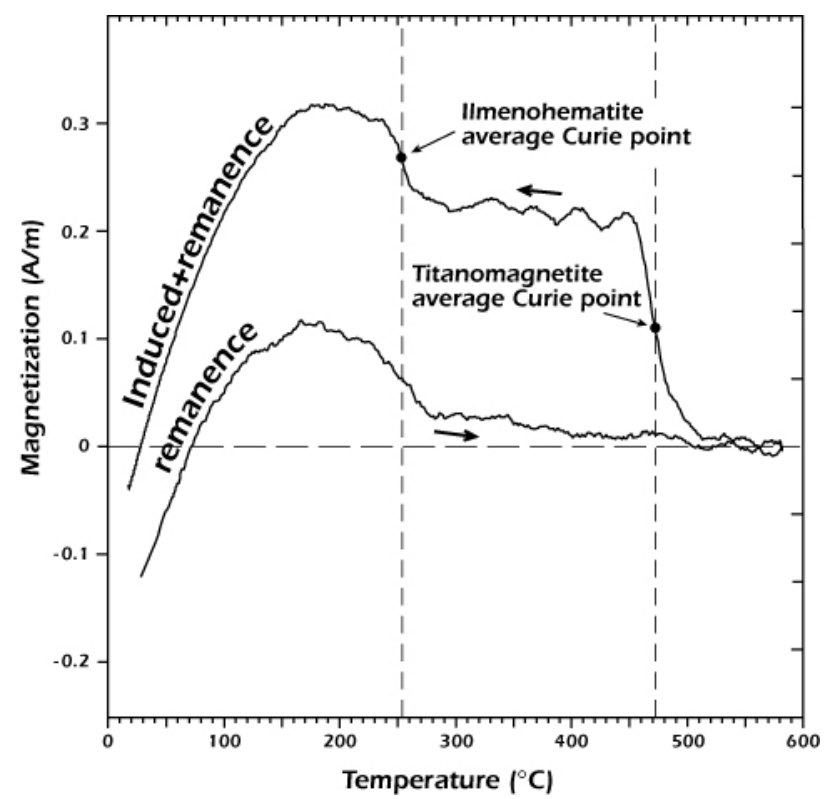

Figure 5. Comparison of the acquisition and demagnetization curves of laboratory TRM $\left(580^{\circ}, 50 \mu \mathrm{T}, \mathrm{T}_{0}\right)$ acquired by Pinatubo rock sample 933 (Bina et al., 1999). The cooling curve was measured under a 50 $\mu \mathrm{T}$ field and thus includes an induced component, while subsequent heating was carried out in zero field. 

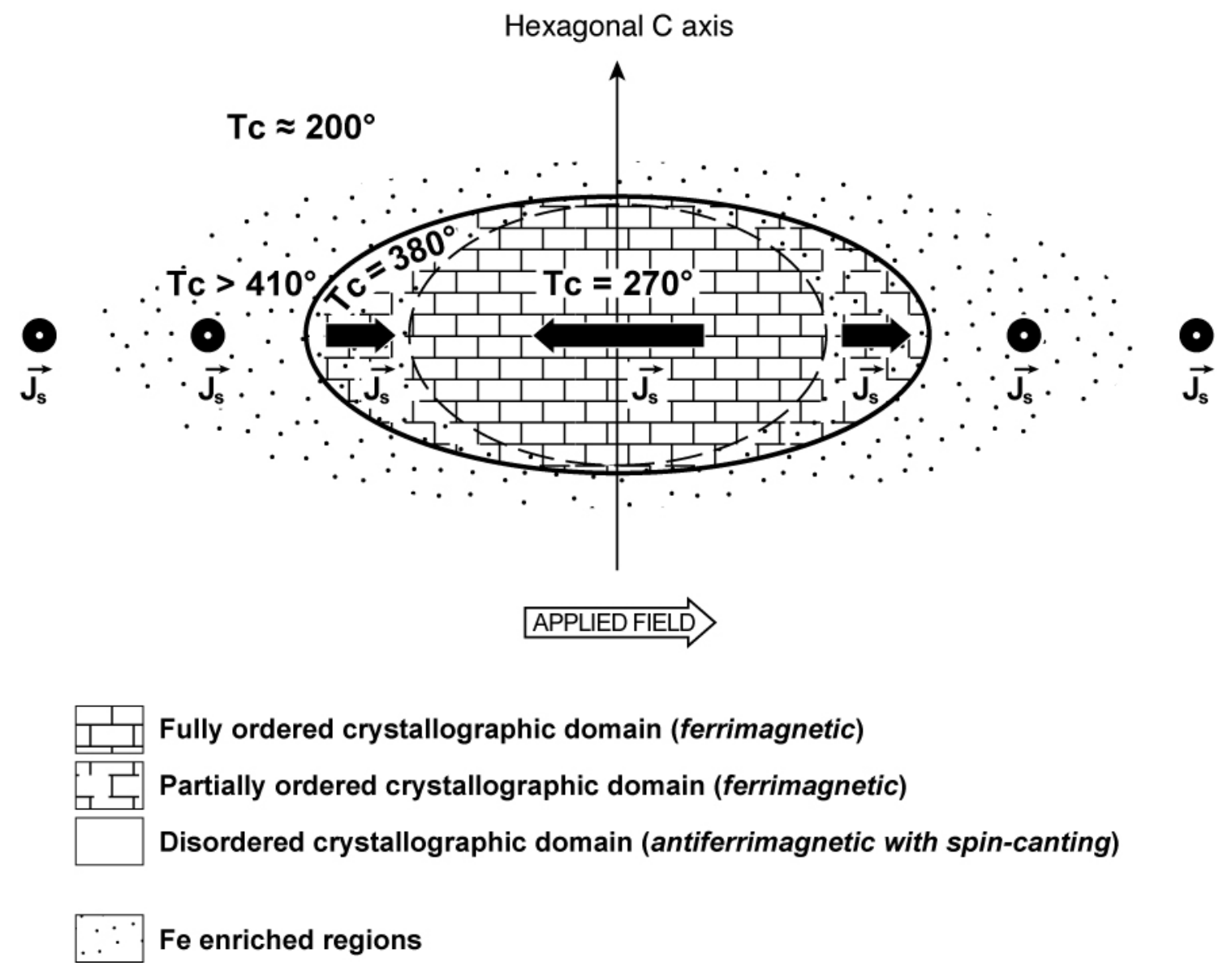

Figure 6. Crystallographic and magnetic model (see text for explanation). The spontaneous magnetization js lies in the basal plane of the hexagonal structure for all mineralogical phases (Goguitchaichvili and Prévot, 2000). However, the js directions in the disordered matrix are at right angle from to those of both the partially (x-phase) and fully (self-reversing) cation-ordered domains. 


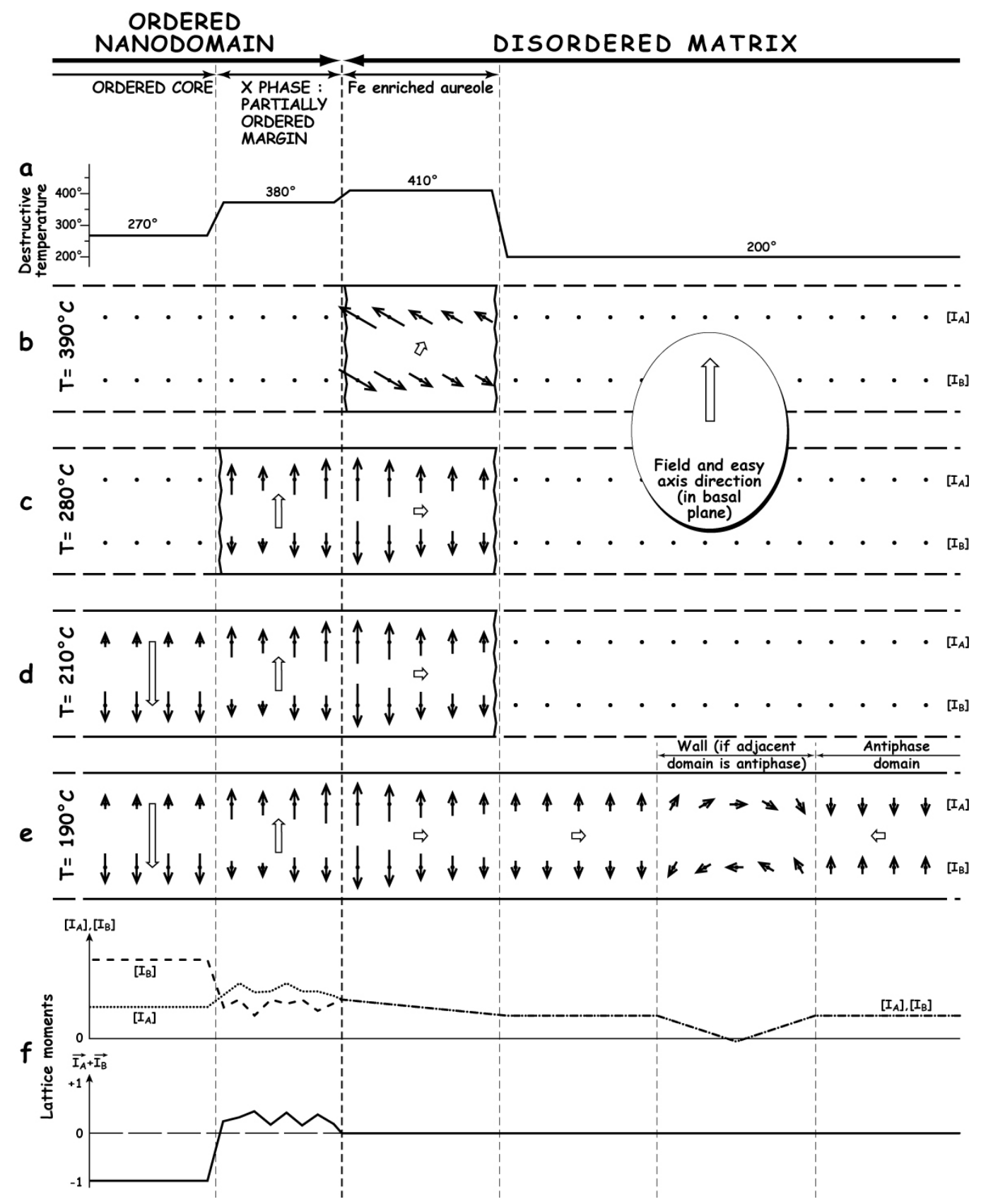

Figure 7. Model of acquisition of self-reversed TRM in a hemoilmenite macrocrystal crystallographically heterogeneous at the nanometer scale (see Fig. 2). (a) : schematic representation of the destructive temperature profile of the crystallographic phases; the destructive temperature corresponds either to the Curie temperature or the blocking temperature of each phase (see text). (b-e) : progressive development of magnetic ordering during cooling; the black thin arrows represent the average magnetic moment of an arbitrary constant number of neighboring atoms in layers A and B; the white arrows represent the whole spontaneous magnetization of the various phases (moments of layers A and B combined); note that all magnetic moments lie in the crystallographic basal plane, which is also the plane of the figure; to be easily identifiable, the moments of layers A and B are conventionally projected onto this plane as two distinct rows; for the sake of simplicity, the applied field direction is taken to be coincident with one of the 3 easy axes of magnetization of macrocrystal. $(f)$ : schematic representation of lattice moments (along the field direction) below $200^{\circ} \mathrm{C}$ (top diagram is unsigned). See text for full description. 